\title{
EchoGéo
}

49 | 2019

Les corridors de développement

\section{Les corridors de développement}

Introduction

Nathalie Fau

\section{(2) OpenEdition}

Journals

Édition électronique

URL : https://journals.openedition.org/echogeo/18098

DOI : 10.4000/echogeo.18098

ISSN : 1963-1197

Éditeur

Pôle de recherche pour l'organisation et la diffusion de l'information géographique (CNRS UMR 8586)

Référence électronique

Nathalie Fau, «Les corridors de développement », EchoGéo [En ligne], 49 | 2019, mis en ligne le 25 octobre 2019, consulté le 10 août 2021. URL : http://journals.openedition.org/echogeo/18098; DOI : https://doi.org/10.4000/echogeo.18098

Ce document a été généré automatiquement le 10 août 2021.

EchoGéo est mis à disposition selon les termes de la licence Creative Commons Attribution - Pas d'Utilisation Commerciale - Pas de Modification 4.0 International (CC BY-NC-ND) 


\title{
Les corridors de développement
}

\author{
Introduction
}

Nathalie Fau

1 Il existe une importante littérature concernant les corridors (Debrie et Comtois, 2010) qui émane aussi bien des scientifiques (géographes, économistes, aménageurs, historiens ou encore sciences du vivant) que des organismes internationaux et régionaux (FMI, banque régionale, Nations Unies). Ce recours à la même notion de corridor pour désigner des réalités si différentes se traduit par des allers retours notionnels entre politique, institutions internationales et milieux scientifiques qui brouillent la compréhension. Les Nations Unies se sont ainsi emparées des concepts géographiques de corridor de transport, défini comme un axe de concentration des infrastructures de communication, et de corridor urbain pour les associer et les décliner sous des formes multiples (Alix, 2012; Debrie et Comtois, 2010; Rodrigue, 2017) : corridor de développement, corridor de commerce ou corridor de croissance. Ces concepts qui se veulent avant tout opérationnels et des outils d'orientation et de programmation des financements servent à désigner un nouveau modèle de développement: les corridors ont pour fonction de favoriser l'implantation de nouvelles activités productives grâce à l'amélioration de l'accessibilité au développement d'infrastructures énergétiques et des capacités de transformation des productions locales et de favoriser le développement des marges et non uniquement des principaux nœuds structurants. Il ne s'agit donc pas seulement de relier les villes entre elles par des axes de communication plus performants, à créer ou améliorer mais de développer un nouveau type d'espace multipolaire et transnational articulant entre elles des régions urbaines existantes et des régions émergentes.

2 Le modèle du «corridor " s'est progressivement imposé sur tous les continents. En 2002, les Nations Unies ont ainsi lancé le projet intitulé Capacity-building in developing interregional land and land-cum-sea transport linkages dont le but est d'identifier dans chacune des régions $\mathrm{du}$ monde les liaisons interrégionales de transport qui contribueraient à une meilleure intégration et favoriseraient le développement économique (ESCAP, 2009). Que ce soit en Amérique Latine, en Afrique ou en Asie, et même en Europe mais selon des modalités un peu différentes, les organisations 
régionales ont ainsi repris et dupliqué un même modèle de développement et d'organisation de l'espace qui a, sinon complétement supplanté ceux existant auparavant, du moins concentré les nouveaux investissements. La figure du corridor a également été adopté par les États, parfois indépendamment de tout cadre institutionnel supranational, soit comme outil d'aménagement de leur propre territoire, soit comme moyen d'affirmation de leur puissance à l'échelle internationale : le projet actuellement le plus ambitieux et le plus médiatique est ainsi celui des " nouvelles routes de la soie » qui doivent relier la Chine au reste du monde grâce à un réseau complexe et multimodal d'infrastructures. Le dossier prend en compte l'ensemble de ces corridors. Soulignons également au préalable que si le terme de corridor de développement est généralement associé à celui d'axe de transport international, dépassant ainsi les limites des frontières nationales, nous avons décidé d'intégrer le texte de Philipe Cadène et d'Yves-Marie Rault traitant des corridors en Inde car dans cet État-continent, fragmenté par de fortes disparités territoriales et divisé en 29 États fédérés disposant d'institutions propres, les problématiques se sont avérées être très similaires.

3 Face à la multiplication des initiatives, des rapports en tous genres, des discours programmatiques qui s'apparentent parfois à de véritables opérations de marketing, ce dossier souhaite apporter une approche critique s'appuyant sur la réalité du terrain et l'analyse méthodique de ces corridors. Il a pour objectif d'interroger de façon comparative les corridors de développement popularisés par les Nations Unies et les grandes institutions régionales et internationales et mis en œuvre tant par ces mêmes institutions et les États que par des opérateurs économiques privés. Son intérêt est ainsi d'avoir pu réunir des contributions qui interrogent la notion de «corridor de développement" à partir de recherches effectuées tant en Europe (G. Carrouet; A.Cariou) qu'en Afrique (V. Fourault-Cauët et J.-F Steck), en Amérique centrale (L. Médina) et en Asie (P. Cadène et Y.-M. Rault ; N. Fau ; T. Foin). Il réside également dans le regroupement de textes questionnant cette problématique aussi bien à des échelles régionales (Amérique centrale, Asie du Sud-Est), que nationales (Inde et Russie) et locales (Chiang Rai en Thaïlande, Nairobi au Kenya et les villes moyenne longeant la LGV Rhin-Rhône).

\section{Définir, délimiter et étudier les corridors}

4 Pour étudier les « corridors ", trois approches ont été privilégiées dans ce numéro.

5 La première adoptée uniquement par Guillaume Carrouet, est de postuler l'existence possible d'un corridor structuré et organisé le long d'un axe de transport, la LGV RhinRhône, sans qu'aucun périmètre institutionnel ne soit officialisé et sans même son inscription dans un découpage institutionnel. Elle présente le grand intérêt de questionner plus explicitement la notion de corridor puisque l'un des objectifs est de déterminer si l'espace étudié peut être appréhendé comme un corridor de développement.

6 La seconde, largement dominante, consiste à centrer son étude sur des corridors prédéfinis et désignés ainsi par des institutions régionales (L. Médina ; N. Fau ; T. Foin) ou des gouvernements nationaux (P. Cadène et Y.-M. Rault; A.Cariou). Ce type d'approche nécessite de s'intéresser au préalable à la genèse de ces corridors afin d'analyser les cadres prescripteurs qui les conçoivent et les raisons de leur émergence. 
Une approche diachronique, "la géohistoire d'un tracé technique " (Debrie, 2007), permet en effet de se rendre compte de l'évolution des discours, des acteurs, des enjeux et des événements qui incitent à la construction de ces corridors (Beyer, 2014; Libourel, 2015). Alain Cariou analyse ainsi les facteurs qui ont incité le gouvernement russe à passer d'une logique nationale où le Transsibérien est pensé uniquement comme un moyen de vaincre l'immensité continentale et d'intégrer les périphéries au territoire national à une logique transnationale où le Transsibérien est envisagé comme un vecteur d'insertion dans la mondialisation, notamment par un rapprochement avec une Asie en pleine croissance, et comme un «instrument de puissance et de développement ». De la même façon, l'émergence en Inde des projets de développement de corridors s'inscrit dans le cadre d'une nouvelle stratégie économique où «le pouvoir central, devenu progressivement favorable au secteur privé, cherche à attirer les capitaux vers ses grands projets » (P. Cadène et Y.-M. Rault). En Amérique centrale (L. Médina), en Asie du Sud-est (N. Fau ; T. Foin), et en Afrique (V. Fourault-Cauët et J.F. Steck), le poids des banques et des institutions régionales ainsi que les stratégies d'intégrations régionales qu'elles impulsent, la recherche d'investissements pour financer les infrastructures et l'orientation vers des politiques économiques extraverties apparaissent déterminantes dans l'adoption de ce modèle de développement. Tous ces projets de corridors sont ainsi élaborés dans le cadre de politique prônant l'ouverture au marché mondial, la libre circulation des échanges et des investissements et les politiques libérales de dérégulation qui l'accompagnent. Soulignons aussi, que la mise en œuvre systématique de ce nouveau modèle de développement peut apparaitre dogmatique tant ces « corridors de développement » ne prennent pas toujours en compte la spécificité des espaces où ils sont implantés. En Asie du Sud-Est, "aucune distinction n'est ainsi faite entre corridor maritime et corridor terrestre ni dans les tracés choisis ni même dans les modalités d'évaluation de leur performance » (N. Fau).

7 Étudier des corridors prédéfinis requiert également de déconstruire les discours des institutions et d'analyser une littérature grise souvent très prolifique et très nombreuse afin de la confronter aux réalités du terrain (Fau et al., 2014). La multiplication des initiatives et des discours programmatiques soulève en premier lieu la question de la faisabilité et de la réalité de ces projets d'intégrations transnationales. Que ce soit en Amérique centrale, en Afrique ou en Asie, les cartes des corridors diffusées par les institutions et abondamment reprises dans les médias ne permettent que rarement de distinguer les projets des infrastructures réellement réalisées, ni même des corridors déjà existants mais selon des modalités différentes. Mesurer la réalité des corridors, c'est ainsi confronter les discours et les objectifs des institutions internationales et des bailleurs de fonds avec les programmes de planification nationale ou locale des États traversés (N. Fau). Cette confrontation systématique permet notamment de mesurer l'implication des différents gouvernements en analysant le degré d'adaptation des politiques de planification nationale aux projets décidés par les bailleurs de fonds. En Asie du Sud-Est, trois types de pays peuvent être distingués (N. Fau) : ceux qui " privilégient, pour des raisons à la fois politiques et économiques, l'amélioration d'une connectivité interne à celle d'une connectivité régionale", ceux qui à l'inverse valorisent le « développement des infrastructures d'intégration régionale sans prendre en compte les logiques spatiales d'aménagement de leur propre territoire » et enfin ceux « qui profitent de cette amélioration de la connectivité régionale pour renforcer leur poids économique tout en développant leurs territoires périphériques ». La 
contribution de Philipe Cadène et d'Yves-Marie Rault montre ainsi ces nombreux décalages entre corridors planifiés et réalités des corridors. En confrontant les plans de développement des corridors définis par le gouvernement indien à leur propre étude cartographique des dynamiques de la répartition du capital productif, les auteurs démontrent non seulement l'existence de corridors industriels dont le dynamisme ne doit rien à cette politique de corridor mais aussi que les corridors planifiés, pourtant dotés d'infrastructures de qualité associées à des zones industrielles, des parcs logistiques et même des villes nouvelles, ne sont pas parvenus à attirer de nouveaux investisseurs. Dans le cas de l'Inde, les logiques d'implantation des entreprises dépendent finalement assez peu des grandes orientations décidées par les autorités publiques.

8 La dernière approche est de confronter les corridors définis par les institutions régionales aux corridors identifiés par les scientifiques. La contribution de Véronique Fourault-Cauët et Jean-Fabien Steck analyse ainsi la manière dont la rhétorique du «corridor» est mobilisée pour légitimer des projets d'aménagement urbain opposés dans la ville de Nairobi au Kenya : aux « corridors de développement " défendus par les pouvoirs publics qui souhaitent faire de Nairobi un carrefour central dans le réseau de corridors de transport de l'Afrique australe et orientale, les scientifiques et environnementalistes opposent le concept de " corridors écologiques » et de «corridor de migrations de la faune sauvage » afin de préserver l'intégrité du parc national de Nairobi menacé par la construction des infrastructures de transport. Dans cette bataille rhétorique, la justification des corridors de développement qui use "d'arguments d'autorité en prenant appui sur des modèles de planification et d'aménagement diffusés par de grandes agences internationales", s'impose face aux discours des scientifiques et des environnementalistes qui s'appuient pourtant sur des travaux et des rapports conséquents. Cette contribution souligne ainsi les «migrations» transdisciplinaires du concept de corridor qui est passé de l'écologie à l'économie et aux sciences du territoire avant d'être récupéré par la Banque mondiale et les Banques régionales. Elle montre également, comme dans le cas des corridors en Asie du Sud Est (N. Fau), la difficulté à s'opposer à la construction de corridors de développement, tant l'imaginaire qu'il porte est imprégné de valeurs positives.

\section{Quel développement le long des corridors ?}

9 Les projets de corridor, souvent qualifiés « de développement » partent souvent du postulat qu'il existe un lien évident entre la construction des infrastructures, le désenclavement des territoires et leur mise en réseau et le développement économique (Steck, 2009 ; Fau, 2016). Ils sont présentés comme des outils de planification favorisant une meilleure insertion dans la mondialisation; leur but est de canaliser les projets d'investissement, de renforcer les processus de croissance économique et de promouvoir et de faciliter le libre-échange. Leurs implantations doivent permettre de réduire le coût des produits importés, d'améliorer l'accès aux marchés internationaux, de faciliter le maillage industriel, d'améliorer l'interdépendance des différents secteurs de l'économie en reliant par exemple davantage les lieux de production avec ceux de consommation. Les corridors auraient donc pour objectifs de favoriser la croissance économique, l'emploi et une amélioration des conditions de vie. Ils suscitent ainsi de nombreux espoirs et attentes auprès des populations locales notamment situées dans 
les espaces périphériques. La majorité des auteurs questionnent ainsi la pertinence et le modèle de développement sous-tendu par ces grands programmes d'infrastructures. Sans pour autant revenir sur le débat sur les effets structurants des transports (Offner, 1993 et les controverses de l'Espace Géographique, 2014), ils cherchent à identifier les mutations territoriales, l'émergence de structures spatiales nouvelles et les retombées sur les espaces traversés par les corridors en adoptant une approche multiscalaire et territoriale.

Les contributions de Lucile Médina et de Alain Cariou soulèvent notamment les difficultés à concilier les impératifs de "fluidité » voulus par les transporteurs et la logistique moderne afin de favoriser les échanges internationaux et celle de "fécondité territoriale ", c'est-à-dire de développement qui bénéficie aux territoires traversés. Cette opposition «fluidité versus fécondité » pour reprendre les termes de Jérôme Lombard et al. (2014), est tangible aussi bien en Russie qu'en Amérique centrale par la prévalence de la fonction de transit des corridors sur celle d'amélioration des conditions de vie de la population. En Amérique centrale, les corridors ont soit pour rôle de relier, sans rupture de charge et le plus rapidement possible, les deux façades océaniques, soit de connecter des enclaves productives aux ports d'exportation et aux marchés extérieurs; ils ne profitent donc souvent qu'aux extrémités portuaires. De façon similaire, Alain Cariou s'interroge sur le " processus vertueux de croissance lié au désenclavement » des territoires par les corridors. Analysant les effets induits par les corridors eurasiatiques et notamment par le Transsibérien, il montre que ces corridors ont pour principale objectif de renforcer les liaisons terrestres entre la Chine et l'Union Européenne et ainsi «qu'entre ces deux pôles de la croissance mondiale, le cœur de l'Eurasie apparaît en creux ». Ainsi, l'amélioration des dessertes de transports a étendu la sphère d'influence des régions économiquement les plus fortes au détriment des zones les plus récemment desservies et à renforcer dans leur fonction les pôles économiques existants. À la conclusion de Lucile Médina «les espaces traversés sont neutres, impensés. Ce qui compte est de les traverser au plus vite ", répond cette autre image de Alain Cariou: "Sur des milliers de kilomètres, les liaisons ferroviaires traversent les territoires vides de la taïga sibérienne, des steppes mongole et kazakhe, les déserts du Xinjiang et du Turkménistan. ». La fonction dominante de ces corridors est ainsi d'être des axes logistiques. Les deux contributions soulignent également le rôle des corridors dans le cantonnement des périphéries à des activités uniquement extractives et à un rôle de fournisseur de matières premières. À propos de la Sibérie, Alain Cariou évoque même "un développement de type colonial » où la fonction de corridor du Transsibérien «se résume à une ligne ferroviaire solitaire qui connecte tout au long du parcours un archipel de villes industrielles vouées au premier traitement des matières premières et à leur exportation. ». La figure du corridor se limitant ainsi à un axe reliant des enclaves d'extraction et d'exportation de matières premières, Alain Cariou en conclut que le Transsibérien ne peut être qualifié de corridor de développement. Ces deux contributions montrent ainsi que si les corridors peuvent intégrer des périphéries, ils peuvent aussi simplement traverser ces territoires (effet tunnel) ou aspirer les ressources de ces régions (effet de pompe). À une échelle plus locale, celle des villes, le risque d'être seulement traversé et non desservi est également manifeste comme le soulignent les contributions de Thomas Foin sur Chiang Rai en Thaïlande et Véronique Fourault-Cauët et Jean-Fabien Steck sur Nairobi. En analysant et en confrontant les recherches menées sur les corridors en Asie du Sud-Est, Nathalie Fau montre par ailleurs que cette opposition entre «fluidité » et « fécondité » 
se reflète également dans les différences de méthodologie mises en œuvre dans les études issues d'une part de la sphère opérationnelle, dominées par les logisticiens dont l'objectif est de supprimer les ruptures de charge qui peuvent entraver cette fluidité, et celles des géographes questionnant davantage les impacts spatiaux et sociaux des corridors.

11 En Inde (P. Cadène et Y.-M. Rault), les corridors ne sont pas parvenus non plus à rééquilibrer un développement économique fortement polarisé par les plus grandes monopoles: "les plus grandes villes indiennes restent au cœur de l'organisation du territoire et rassemblent, pour la presque totalité des secteurs industriels, l'essentiel des capacités de contrôle et des investissements productifs. ». Ils ont même contribué à accentuer la domination économique des plus grands centres urbains en favorisant notamment le "quadrilatère doré » formé par Delhi, Kolkata, Chennai, Bangalore et Mumbai et les grandes régions qu'elles commandent. Le seul changement majeur est une diffusion du capital productif à partir des grands centres urbains le long des axes de transports aboutissant davantage à la formation de corridors industriels que de corridors de développement. Les auteurs montrent également que des cinq corridors de développement promus par l'État, seulement trois se sont transformés en corridors industriels tandis que ces derniers "sont plus nombreux que ceux projetés et encouragés par l'État central».

12 À l'inverse, les contributions de ce numéro sur l'Asie du Sud-Est et l'Europe soulignent le rôle des corridors dans l'émergence de nouvelles polarités. En modifiant les possibilités d'accessibilité, les corridors contribuent à la requalification des territoires, notamment des périphéries nationales qui deviennent de nouvelles centralités au sein de réseaux d'échanges transnationaux. Dans le cadre de l'espace Rhin-Rhône (G. Carrouet), la construction d'une ligne à grande vitesse a contribué à la valorisation de villes moyennes: «Bien que conçue pour répondre aux besoins de transports des grands foyers urbains qui l'entourent, la LGV apporte un surplus d'accessibilité non négligeable aux agglomérations moyennes ». Le fait est assez exceptionnel en France où la construction d'une infrastructure à grande vitesse entre des grands pôles engendre généralement " un effet tunnel », c'est-à-dire une perte d'accessibilité relative pour les pôles secondaires. À une échelle plus locale, les transformations territoriales induites par la construction de la LGV se concentrent aux abords des gares et prennent la forme de nouveaux pôles d'activités tertiaires : immeubles de bureaux, centres d'affaires et infrastructures hôtelières.

Les études sur l'Asie du Sud-Est montrent que les corridors ont modifié les hiérarchies urbaines régionales et favorisé l'émergence de trois nouveaux types de nœuds structurant le fonctionnement interne des corridors: les têtes de corridors et de réseaux, les zones frontalières et les espaces intérieurs situés aux croisements des corridors (N. Fau). L'intérêt majeur de la contribution de Thomas Foin est d'analyser les dynamiques sociales et économiques induites par les corridors à une échelle locale, celle de la ville. Il réside également dans le choix, non d'une grande agglomération ou d'une tête de corridor, mais d'une ville moyenne, Chiang Rai, située dans une province périphérique frontalière de la Thaïlande et sur un corridor méridien majeur structurant la Région du Grand Mékong et reliant Bangkok à Kunming. Pour mesurer l'impact de ce corridor transnational sur le développement urbain, l'auteur étudie les marqueurs et les processus de l'internationalisation : mutations du bâti et des secteurs économiques. Cette approche lui permet de démontrer une spécialisation des lieux et 
une modification de la configuration urbaine marquée notamment par l'émergence de nouveaux pôles (touristique, financier et commercial) et une augmentation du foncier le long du corridor. La situation de Chiang Rai sur un corridor induit également une reconfiguration des hiérarchies urbaines : la ville concurrence désormais directement Chiang Mai, la capitale économique et politique du Nord de la Thaïlande, en exerçant notamment une nouvelle fonction de commandement sur les villes frontalières de Mae Sai, Chiang Saen et Chiang Khong.

La majorité des contributions de ce numéro souligne cependant l'absence de la prise en compte des questions environnementales dans les projets de planification des corridors. Le long du Transsibérien, les terres sont polluées par le rejet des mines mais aucun contrôle n'est effectué sur le respect des réglementations environnementales (A. Cariou); en Amérique centrale, aucune étude d'impact environnemental n'est envisagée en amont de la construction des corridors en dépit d'une mobilisation et d'une opposition de plus en plus importante des scientifiques, des organisations de défense de l'environnement et de la société civile (L. Médina). En Thaïlande, les projets de planification de Chiang Rai n'ont que très récemment pris en compte les dégradations environnementales induites par le corridor (T. Foin). Cette question, de la difficulté à concilier développement économique et protection de l'environnement, est au cœur de l'article de Véronique Fourault-Cauët et Jean-Fabien Steck. À partir d'une analyse des principaux documents de planification impliquant Nairobi, les auteurs démontrent l'absence d'une prise en compte environnementale dans la planification urbaine de la ville; les enjeux de développement nationaux liés à la construction du Northen Corridor l'emportent en effet très largement sur ceux de la préservation du parc de Nairobi et de la savane environnante. Dans le cadre de la métropolisation de Nairobi, le parc apparait même davantage comme une contrainte dans la mise en place d'un corridor «du fait de son statut foncier a priori inaliénable» qu'un atout pour la promotion d'un développement durable tel qu'il est pourtant préconisé dans l'Agenda 2063 de l'Union Africaine ou de l'African Urban Agenda Programme. Et les auteurs de conclure : « ce corridor de développement peut difficilement être qualifié de durable, et met $a$ contrario en évidence la fragilité d'une notion qui occulte largement la spécificité des espaces traversés par les axes de transport, ainsi que leurs conséquences environnementales et sociales ".

\section{La mise en œuvre des corridors, une nouvelle forme de gouvernance?}

15 Les corridors sont envisagés comme de nouveaux outils de gouvernance (Priemus et Zonneveled, 2003). L'action publique des États est complexifiée avec non seulement la nécessité de multiplier les accords bilatéraux ou multilatéraux mais aussi avec celle de prendre en compte d'une part le secteur privé qui finance et exploite ces corridors et d'autre part les autorités locales dont le pouvoir s'est accru avec les mouvements de décentralisation. La gestion des corridors transnationaux impose un nouveau cadre de gouvernance, impliquant la mise en place d'une collaboration avec une multitude d'acteurs et "repose sur une forme de coordination entre chaque composante territoriale et économique" (Debrie et Comtois, 2010). Cette question de la gouvernance se pose à toutes les étapes de mise en place du corridor : décision du tracé, mode de financement et enfin gestion et fonctionnement. 

formes de gouvernance. Véronique Fourault-Cauët et J.ean-Fabien Steck montrent ainsi que dans le cadre de l'implantation du Nothern Corridor soutenue par l'Union Africaine et renforçant la fonction de carrefour logistique de Nairobi, les enjeux internationaux et nationaux priment sur les enjeux locaux et excluent en revanche les autorités métropolitaines de Nairobi des processus de décisions concernant le tracé du corridor qui empiète pourtant sur le parc de Nairobi ; ce parc, concluent les auteurs « apparaît dans un entre-deux mal défini, dans lequel les dispositifs de concertation et de gouvernance locales récemment mis en place sont encore peu efficients face au pouvoir central ». Ce manque de concertation entre les acteurs empêche la mise en place d'un compromis conciliant les préoccupations de développement et de conservation de l'environnement. En Russie, le faible impact des corridors sur les espaces traversés s'explique en grande partie par l'absence de concertation et de coopération entre l'État et les autorités locales: "Le développement est une affaire d'État, planifié par un pouvoir central autoritaire, de sorte que toute forme locale d'autonomie politique et d'action entrepreneuriale est annihilée " (A. Cariou). De même en Inde, le succès très relatif des corridors de développement planifiés par l'État indien s'explique en partie par le manque de coordination entre les politiques de l'État fédéral et des États fédérés même si une forme de gouvernance, jusqu'alors inédite en Inde, émerge dans le cadre d'une coopération l'État central, les pouvoirs publics et les milieux entrepreneuriaux (P. Cadène et Y.-M. Rault).

17 Cette question de la gouvernance est au cœur de la contribution de Guillaume Carrouet. L'auteur compare les organisations et les coopérations territoriales mises en place par les acteurs locaux pour obtenir la desserte d'une ligne à grande vitesse à celles instaurées huit ans après l'ouverture de la première phase de la LGV Rhin-Rhône. La formation du réseau de villes Métropoles Rhin-Rhône avait ainsi pour objectif de formaliser un système de gouvernance reposant sur la coopération entre des territoires desservis par une même infrastructure de transport. L'auteur démontre que si ce mode de gouvernance fonctionnait relativement bien dans le cadre d'une coalition de projets visant à faire pression sur le gouvernement et les institutions européennes afin d'obtenir la grande vitesse ferroviaire et peser sur la délimitation du tracé, il est devenu en revanche inopérant une fois la construction du LGV effectuée. L'échec de cette gouvernance territoriale résulte de "l'effacement de la coalition de projet au profit de l'intérêt porté par chaque collectivité à la desserte de son propre territoire » ; il est ainsi tangible par la multiplication aux abords des gares de projets d'aménagement similaires et concurrents pensés sans réel souci de développer une complémentarité entre les territoires. La gouvernance au sein de la Métropole RhinRhône s'avère ainsi davantage « un concept théorique que pratique ». Et l'auteur d'en conclure que si l'espace Rhin-Rhône a été un temps un territoire de projets, il ne peut être qualifié de corridor de développement du fait de l'absence d'une gouvernance régionale.

La question de gouvernance se pose également dans le financement des corridors. Le modèle le plus fréquemment choisi est un partenariat public-privé (cas tant de l'Amérique centrale, que de l'Inde, de l'Afrique et de l'Asie du Sud-Est). S'il apparait comme une solution au manque de possibilités de financement de l'État, il suscite également de nouveaux problèmes et inquiétudes. Le premier concerne le manque de continuité dans la réalisation des infrastructures : en Amérique centrale dont l'opacité 
des financements est soulignée par Lucile Médina, la plupart des projets sont inachevés, soit en raison de la faillite de l'investisseur principal, soit du fait de la volatilité des investissements. Le second est celui de la souveraineté nationale et d'intégration nationale : comment concilier les objectifs et les intérêts souvent très divergents entre État, opérateurs privés du secteur de la logistique et des transports et investisseurs? Les infrastructures étant l'outil et le reflet de la puissance d'un État, comment envisager que celles-ci deviennent la propriété d'un État tiers ou d'un opérateur économique privé entièrement libre d'en concevoir le développement (Lombard et al., 2014) ? Le financement est ainsi parfois accompagné d'accords de concession à long terme non seulement sur l'exploitation des infrastructures de transport mais aussi sur les terres que le corridor traverse. Un parallèle peut être effectué entre le Grand Canal du Nicaragua et les corridors qui traversent la Birmanie (N. Fau et L. Médina). Dans ces deux projets financés majoritairement par la Chine l'organisation spatiale du territoire national est déstabilisée au profit d'acteurs extérieurs : le canal du Nicaragua divise ainsi physiquement le pays sans qu'aucune mesure de franchissement ne soit envisagée et quant à la Birmanie, les connexions internationales sont privilégiées au détriment des axes et organisations spatiales nationaux.

\section{Des outils d'intégration régionale ou d'affirmation de puissance?}

Les corridors sont également conçus comme des outils «des politiques d'intégration régionale visant à fluidifier et intensifier les échanges entre les espaces nationaux " (Debrie, 2018). Transcendant les frontières physiques, politiques, administratives, sociales et économiques, ils devraient favoriser l'interdépendance d'espaces situés dans des territoires nationaux différents. Les organisations régionales présentent ainsi les corridors comme le support des projets d'intégration économique et elles œuvrent afin que la construction des infrastructures aillent de pair avec une modification des législations nationales permettant de supprimer les discontinuités frontalières, qu'elles soient administratives, techniques, juridiques ou encore tarifaires. L'objectif est de favoriser les échanges économiques au sein d'un marché efficace car intégré. Il est également de programmer, de hiérarchiser et de flécher les financements des infrastructures de transports (Debrie, 2018). Les corridors sont ainsi envisagés comme un outil de planification régionale en appui des processus de rapprochements entre États. Comme le souligne Jérôme Lombard et Nora Marei (2018), "ces projets d'infrastructures dessinent des espaces régionaux d'échanges qui traduisent la volonté d'unifier les normes, les services et les marchés ».

Les corridors sont-ils ainsi vecteurs et porteurs d'intégration régionale? Cette question est récurrente dans les contributions s'intéressant au processus de formation de macrorégion et à l'interdépendance des territoires nationaux. Dans le cadre de ce numéro, les auteurs insistent surtout sur les obstacles qui empêchent ces corridors de devenir pleinement des outils d'intégration régionale. Le plus difficile à surmonter semble être le refus, au minimum implicite, des gouvernements nationaux à penser l'organisation de l'espace au-delà de l'échelle de leur propre État et à soutenir des projets transnationaux qui risquent de renforcer les concurrences entre les territoires. En Amérique centrale en dépit d'une volonté affichée de favoriser l'intégration régionale, la multiplication des projets de corridors "vient contredire le discours et trahit 
l'absence d'une vision régionale concertée » (L. Médina). Chaque État met en avant son propre projet de corridor afin d'en tirer les dividendes sans envisager la construction d'un corridor réellement inclusif à l'échelle régionale. Lucile Médina montre par ailleurs que ces initiatives étatiques fragmentées ne permettent pas de planifier un corridor interocéanique entre les deux façades maritimes Atlantique et Pacifique capable de rivaliser avec d'une part le canal de Panama et d'autre part les ponts terrestres transocéaniques des États-Unis et ceux émergeant au Mexique. Faute d'accords et de concertations régionales, le « rêve » des gouvernements de valoriser la situation de leur territoire sur l'isthme centraméricain en le transformant en " une plateforme multimodale stratégique dans le système mondial de transport de marchandises » a peu de chances de se réaliser. De même, Alain Cariou, s'interrogeant sur les réticences et le peu d'empressement du gouvernement russe à moderniser les interconnexions du Transsibérien avec le réseau chinois, analyse le dilemme de la politique du gouvernement «prise entre la tentation d'intégrer son territoire à l'espace-monde afin de retrouver son rang mondial tout en le préservant d'une trop grande ouverture à l'égard de ses voisins, notamment chinois.». Pourquoi favoriser l'ouverture de corridors transnationaux s'ils profitent surtout aux pays limitrophes? En Asie du Sud-Est, il n'est ainsi en rien surprenant que le gouvernement thaillandais soit le principal pays à promouvoir les corridors: en position de carrefours des corridors et économiquement plus développés que ses voisins, la Thaïlande en est le principal bénéficiaire. En revanche, en Indonésie, le gouvernement freine le raccordement du territoire indonésien aux réseaux transnationaux afin de protéger une économie encore trop fragile pour être confrontée à la concurrence (N. Fau).

Se focaliser sur les corridors pour étudier les processus d'intégration régionale permet aussi d'interroger le découpage des régions institutionnelles et de faire apparaitre les projets concurrents de régionalisation : des corridors concurrents peuvent en effet être porteurs de projets d'intégrations régionales différents. Les projets de corridors mis en place par la Chine en Asie du Sud-Est vont ainsi à l'encontre du plan de connectivité de l'ASEAN; ils pourraient être porteurs d'une dissolution de l'ASEAN entre sa partie continentale et insulaire et favoriser l'émergence d'une nouvelle région non institutionnalisée centrée sur la Chine et ses voisins limitrophes (N. Fau). En Russie, la construction des corridors est à l'origine d'un rapprochement entre la Sibérie et l'Asie Orientale (Chine, Japon, Corée du Sud) et de l'émergence d'un processus de régionalisation transnationale (A. Cariou). Les projets de corridors de plus en plus nombreux et souvent en concurrence les uns avec les autres, s'inscrivent ainsi dans le cadre d'une géopolitique régionale. L'Amérique centrale (L. Médina) est ainsi au cœur d'une rivalité entre les investisseurs chinois (projet du creusement du Grand Canal de Panama) et américains (Plan Puebla-Panama). En Asie centrale et en Mongolie, le contrôle, l'exploitation et le financement des nombreux corridors, désormais en concurrence pour capter les flux transnationaux, sont devenus un enjeu d'influence et de pouvoir entre la Russie et la Chine. De fait, on peut se demander si la multiplication de ces corridors transnationaux sera en mesure de donner une existence et une réalité concrète à un "espace eurasiatique » (A. Cariou). En Europe (G. Carrouet), l'espace Rhin-Rhône n'est qu'un " corridor imparfait » car sa dynamique d'interface entre Mer du Nord et Mer Méditerranée est remise en cause par «la présence de maillons concurrents à l'échelle européenne dont le corridor Rhin-Alpin ». Le choix du tracé des corridors est ainsi le reflet de négociations et de luttes d'influence et le terme de " connectivité » utilisé par les institutions régionales et les bailleurs de fonds vise 
souvent par l'utilisation d'un vocabulaire techniciste et par souci d'un discours consensuel à masquer cette réalité (N. Fau).

Centrer un dossier sur «le corridor de développement » soulève ainsi la difficulté à définir cette notion et les espaces qui lui sont associés. Est-ce qu'un simple axe de transport peut être qualifié de corridor? Dans leur contribution, les auteurs répondent par la négative en montrant que pour être qualifié ainsi, celui-ci doit induire non seulement une dynamique de croissance économique et de développement territorial des espaces traversés mais aussi l'émergence de nouvelles formes de gouvernance et d'intégration spatiale. De fait, parmi les corridors étudiés presqu'aucun n'est validé comme corridor de développement. L'intérêt est ailleurs. Il est de permettre des comparaisons et d'inciter à la mise en place d'une méthodologie et d'une grille d'analyses communes pour que les géographes s'emparent de cette notion de corridor de développement et confrontent leur approche à celles opérationnelles et programmatiques. Analyser ainsi les «nouvelles routes de la soie» promues par la Chine qui s'étendent désormais sur tous les continents, permettrait de mieux en mesurer la réalité et ses impacts.

\section{BIBLIOGRAPHIE}

Alix Y. (dir.), 2012. Les corridors de transport. Cormelles-le-Royal, Editions Ems, 344 p.

Bender S., 2001. Trade Corridors: The Emerging Regional Development Planing Unit in Latin America. In Edginton D., Fernandez A., Hoshino C. (ed.), New Regional Development Paradigms, vol. 2. United Nations Centre for Regional Development, Greenwood Press, p. 39-56.

Beyer A., 2014. La construction d'un espace européen des transports. Du principe de souveraineté nationale aux modalités de son dépassement. HDR Géographie, Université de Paris IV Sorbonne, $320 \mathrm{p}$.

Debrie J., 2007. Géohistoire d'un tracé technique : frontières et réseaux en Afrique de l'Ouest continentale « francophone ». Flux, vol. 4, n 70, p. 49-58.

Debrie J., 2018. Corridor : un outil de l'intégration régionale ? In Mareï N., Richard Y., Dictionnaire de la régionalisation du monde. Paris, Khartala, p. 118-119.

Debrie J., Comtois C., 2010. Une relecture du concept de corridors de transport : illustration comparée Europe/Amérique du Nord. Les Cahiers Scientifiques du Transport, n 58, p. 127-144.

Economic and Social Commission for Asia and the Pacific (ESCAP), 2008. Joint study on developing euro-asia transport linkage.

Fau N., 2016. Investment in infrastructure and regional integration: Will connectivity reduce inequalities? In Br. Jetin B., M. Miki (ed.), ASEAN Economic Community: A model for Asia-wide Regional Integration? Palgrave Macmillan, p. 291-310.

Fau N., Taillard T., Khonthapane S., 2014. Transnational Dynamics and Territorial Redefinitions in Southeast Asia: the Greater Mekong Subregion and Malacca Strait economic corridors. ISEAS, Institute of South-East Asia à Singapour, $517 \mathrm{p}$. 
Les controverses de l'Espace Géographique, 2014. Les effets structurants des infrastructures de transport. L'Espace géographique [En ligne], tome 43, n 1, p. 51-67. https://www.cairn.info/revueespace-geographique-2014-1-page-51.htm

Libourel E., 2015. Le corridor ferroviaire méditerranéen : planification, politisation et territorialisation d'un projet d'aménagement. Architecture, aménagement de l'espace. Doctorat, Université Paris-Est.

Lombard J., Ninot O., Steck B., 2014. Corridors de transport en Afrique et intégration territorialesen questions. In Gana A., Richard Y. (dir.), La régionalisation du monde : construction territoriale et articulation global-local. Tunis-Paris, IRMC-Karthala, p. 253-272.

Lombard J., Mareï N., 2018. Infrastructures et transports : puissants facteurs d'intégration régionale. In Mareï N., Richard Y. (dir.), Dictionnaire de la régionalisation du monde. Paris, Khartala, p. $168-169$.

Offner J.M, 1993. Les effets structurants du transport : mythe politique, mystification scientifique. L'Espace géographique, tome 22, $\mathrm{n}^{\circ}$ 3, p. 233-242.

Priemus H., Zonneveled W., 2003. What are corridors and what are the issues? Introduction to special issue: the governance of corridor. Journal of transport geography, vol. 11, p. 167-177.

Raffestin C., 1980. Pour une géographie du pouvoir. Paris, Librairies techniques, 249 p.

Rimmer P.J., 2004. Global Flows, Local Hubs, Platforms and Corridors: Regional and Economic Integration in Northeast Asia. Journal of International Logistics and Trade, vol. 1, n 2, p. 1-18.

Rodrigue J-P, 2017. The Geography of Transport Systems. New York, Routledge, 440 p.

Steck B., 2017. Entre fermeture fantasmée et ouverture effective : les infrastructures de transport, levier majeur du développement de l'Afrique. Revue internationale et stratégique, $\mathrm{n}^{\circ} 107$, p. 145-153.

Steck B., 2009. Transport et développement. In Brocard M., Transports et territoires. Enjeux et débats. Ellipses.

\section{INDEX}

Thèmes : Sur le Champ - Sur le Terrain

\section{AUTEUR}

\section{NATHALIE FAU}

Nathalie Fau, Nathalie.Fau@univ-paris-diderot.fr, est maître de conférences à l'Université Paris 7 Denis Diderot, Sorbonne Paris Cité, membre de l'UMR 245 CESSMA et membre du projet de recherche européen Competing Regional Integrations in Southeast Asia (CRISEA) piloté par l'École française d'Extrême-Orient. Elle a récemment publié :

- Fau N., Franck M., 2019. L'Asie du Sud-Est. Émergence d'une région, mutation des territoires. Armand Colin, $434 \mathrm{p}$.

- Fau N., de Trégoldé B., 2018. Mers d'Asie du sud-est. Coopérations, intégrations et sécurités. CNRS édition, $394 \mathrm{p}$.

- Delfolie D., Fau N., Lafaye de Micheau E., 2016. La Malaisie et la Chine, une relation précieuse. Carnet de l'IRASEC n 7, Série Enquête, 272 p. [En ligne]. https://books.openedition.org/irasec/101 